\title{
A free-standing space elevator structure: a practical alternative to the space tether
}

\author{
B. M. Quine $e^{\mathrm{a}, \mathrm{b}}$, R. K. Seth ${ }^{\mathrm{b}}$ and Z. H. Zhu ${ }^{\mathrm{a}}$ \\ ${ }^{\mathrm{a}}$ Department of Earth and Space Science and Engineering, York University, 4700 Keele \\ Street, Toronto, Ontario, Canada M3J 1P3. \\ ${ }^{\mathrm{b}}$ Department of Physics and Astronomy, York University \\ 4700 Keele Street, Toronto, Ontario, Canada M3J 1P3.
}

\begin{abstract}
Space tethers have been investigated widely as a means to provide easy access to space. However, the design and construction of such a device presents significant unsolved technological challenges. We propose an alternative approach to the construction of a space elevator that utilises a free-standing core structure to provide access to near space regions and to reduce the cost of space launch. The structure is comprised of pneumatically inflated sections that are actively controlled and stabilised to balance external disturbances and support the structure. Such an approach avoids problems associated with a space tether including material strength constraints, the need for inspace construction, the fabrication of a cable at least 50,000 km in length, and the ageing and meteorite-damage effects associated with a thin tether or cable in Low Earth Orbit. An example structure constructed at $5 \mathrm{~km}$ altitude and extending to $20 \mathrm{~km}$ above sea level is described. The stability and control of the structure, methods for construction and its utility for space launch and other applications are discussed.
\end{abstract}

Keywords: Space elevator, cantilevered beam, pneumatic structure.

\section{Introduction}

To access space or near space, payloads must gain significant potential and kinetic energy. Traditionally, regions above aircraft altitude are accessed using rocketry, where mass is expelled at high velocity in order to achieve thrust in the opposite direction. This 
process is extremely inefficient as rockets must counter the gravitational force during the flight by carrying mass in the form of propellant and must overcome atmospheric drag. In contrast, if a payload is at least partially hauled to space or near space along an elevator system, the work done is significantly less as no expulsion mass must be carried to do work against gravity, and lower ascent speeds in the atmosphere can virtually eliminate atmospheric drag. Elevator cars' motion may also be powered remotely by electrical or inductive means, eliminating the need to carry any fuel. Stations can be provided that are stationary in space and, consequently, leverage the advantages offered by geostationary orbit but from a vantage point both closer and physically connected to the surface.

In this paper, we describe the design concept for a space elevator based on a selfsupporting core structure that is pressurised pneumatically and controlled actively in order to support the structural load and dampen external disturbance forces.

\section{Background}

It has previously been proposed, most famously by Arthur C. Clarke in his 1978 novel, The Fountains of Paradise, that a space elevator could be constructed using a cable and counter-balanced mass system [1]. For Earth's gravity and spin rate, such a solution requires a cable of at least $35,000 \mathrm{~km}$ in length and a counter balance mass similar to a small asteroid. Such a system could be constructed by launching the cable into space or manufacturing it in situ and lowering it into contact with Earth. However, the technological obstacles that must be overcome, including the construction of a cable with suitable strength characteristics or the in-space construction of the apparatus, have not been realized since Clarke popularized the concept. Known materials are simply not strong enough to enable the construction of a cable of that length that would even be capable of supporting its own weight.

Pearson (1975) provided a physical basis for the construction of such a device [2]. Along with Clarke, he reasoned that the tower must be constructed from geostationary orbit outwards in both directions in order to keep the structure in gravitational balance. Using force derivatives, he calculated that the tower or cable would need to be at least $144,000 \mathrm{~km}$ long in order to be in balance with a net weight of zero and characteristic of 
the Earth's radius, surface gravity, and period of rotation. He proposed an area taper exponent for the structure of $0.776 r_{0} / h$ where $r_{0}$ is the radius of the Earth and $h$ is the specific strength or characteristic height to which a constant diameter tower could be built to in a $1 g$ field. Suitable materials can be classified from the relation $h=\sigma / \rho g_{0}$ where $\sigma$ is the maximum allowable material stress and $g_{0}$ is the surface gravity. Pearson proposed graphite crystals with a material stress of $46.5 \mathrm{GPa}$ and density $2200 \mathrm{kgm}^{-3}$ yield a solution with $h=2150 \mathrm{~km}$ and a taper ratio of 10 without safety factors applied. Such a structure would certainly have significant utility; payloads ascending to the top of the structure could even be injected into escape orbits without the use of rocketry. However, the construction of the device, requiring innovations in materials and in space manufacture as well as at least 24,000 flights of a modified space shuttle with geostationary capability to raise the construction material, place severe constraints regarding practicality.

Edwards (2000) argues that a counter balance comprising spent upper rocket stages (3440 kg/stage) would reduce the cable length to 117,000 km and proposes a ribbon-like structure comprising $1.5 \mu \mathrm{m}$ ribbons each of mass $5000 \mathrm{~kg}$ [3]. Assuming a structure comprising carbon-nanotubes (of density $1300 \mathrm{kgm}^{-3}$ and tensile strength $130 \mathrm{GPa}$ ) with epoxy composite sections, then four Titan IV/Centaur launches would be required to deliver to GEO the initial ribbons. These would be capable of sustaining a climbing robot of mass $528 \mathrm{~kg}$ with a safety margin of two. Climbers would then haul up and attach with epoxy further nanotube tapes, each contributing $7.96 \mathrm{~kg}$ to the load capacity of cable and using an average of $42 \mathrm{~kW}$ of electrical power beamed to the climber by a $4 \mathrm{GW}$ microwave or laser groundstation. After 250 ascents, the cable would be capable of raising a 13,000 kg payload every five days. This concept is seemly much more practical than Pearson's in-orbit construction approach. However, as Edwards identifies, there are other engineering challenges that would seem to place severe constraints regarding the practicality of such a device:

1. Meteorite damage will destroy Earth-to-space cables of dimension less than several centimeters within weeks. Construction is therefore a race against time and the completed cable would need continuous maintenance and repair. The climbers would 
need to be able to ascend through a wide variety of partially severed cable conditions without inducing further failure. Based on the ribbon width, Edwards estimates the probability of a meteor's severing one or more of the initial cables at 0.4 per year. However, the analysis would seem to imply that meteors would strike the cable perpendicular to the width dimension rather than at some acute angle that would cause much smaller meteorites to induce ribbon failure.

2. Low Earth Orbit (LEO) cable impacts from natural and artificial satellites $10 \mathrm{~cm}$ or larger would be expected to occur at a rate of 1 impact per year. Consequently, a mechanism to maneuver the cable (perhaps by moving the anchor point) would be required, and a high-accuracy radar tracking program would be needed to map precisely the orbital trajectories of objects intersecting the cable.

3. Atomic oxygen damage will remove epoxy/nanotube material at a rate of approximately $1 \mu \mathrm{m} /$ month. Consequently, a surface coating would be required to protect the cable at altitudes with high atomic oxygen densities. The coating would need to be resistive to mechanical abrasion (from the climbers) and would also likely require reapplication during the lifecycle of the device.

4. Lightning strikes would pose a significant risk to cable integrity. The construction of the anchor point at high altitude would reduce the probability of a strike; however, the probability of a nearby strike is estimated at 1 every 13 years based on data gathered in Alaska. This figure seems unacceptably high given that a single strike is likely to severe the cable entirely. Furthermore, the lightening data does not account for the increase in lightening frequency due to the presence of the cable itself, and, consequently, the actual risk is highly uncertain. It seems unlikely that this problem can be easily mitigated.

Since the original concept was popularized, several alternative elevator concepts have been proposed. In 2000, Boyd and Thomas proposed a design for a space elevator that moves payloads between locations located at substantially fixed orbital distances from the Earth [4]. The device incorporates a cable system capable of transporting payloads between orbital locations leveraging the energy efficiency of an elevator device that 
climbs the tether. As the device has no part attached to the Earth's surface, a secondary means must be utilized to attach initially the payload to the elevator.

In 2004, Dempsey proposed a system and method for a space elevator comprising a flexible tension structure deployed above and below geosynchronous altitude [5]. In contrast to the traditional tether approach, Dempsey proposes the use of a transport tether shaped into double catenary with one catenary below synchronous orbit altitude and the second catenary above synchronous orbit altitude. The tether configuration forms a harmonic oscillator using a combination of gravitational and centripetal forces with the zero crossing of the harmonic oscillator at an altitude of approximately one half synchronous orbit altitude of the attached elevator. One end of the tether is substantially attached to an equatorial surface location enabling transportation from the surface. The other end of the tether is attached to a counter mass in an orbit above geosynchronous altitude. The tether is configured such that it extends down from geosynchronous orbit to near the ground, loops back up to geosynchronous altitude and finally loops back down to the surface mounting point. An additional tether attached to the surface is utilized to stabilize the downward loop and for control of the elevator. Payloads may be raised using centripetal force in an energy-efficient manner without the need for additional power sources; however, the method requires a cable substantially longer than Clarke's original concept and, consequently, is subject to an even greater engineering challenge to manufacture a suitable tether.

All such structures rely on the development of materials to construct cables of enormous strength. The most common approach is the application of carbon-nanotube; however, Pugno (2006) argues that the presence of microscale defects alone will prevent the fabrication of a cable with sufficient tensile strength even if the theoretical strength limit can be realized in a macroscopic cable [6]. Pugno concludes that a geosynchronous space tether, if built as designed today, will certainly break.

Another intriguing proposal for a space elevator is the space fountain. In this concept, a cable-like structure is constructed that guides a high-speed mass stream in an evacuated closed loop, usually by superconducting magnets. The cable is supported by momentum exchange with the mass stream. A ground-based station reaccelerates the mass stream to 
compensate for losses. A space station attached at the top of the cable maintains a geostationary position without the need of geosynchronous orbit.

Lofstrom (1985) describes launch loop -a $2000 \mathrm{~km}$ cable structure anchored at both ends and at intervals along its length that extends $80 \mathrm{~km}$ vertically [7]. The structure is maintained by energy and momentum exchange with a moving ribbon of mass $15.6 \times 10^{7} \mathrm{~kg}$, which is accelerated to $14 \mathrm{kms}^{-1}$ inside the cable. The ribbon is also used for payload acceleration. We estimate the mass of the structure as $1.5 \times 10^{7} \mathrm{~kg}$ from the data provided and assuming a single ribbon system. The main disadvantage of the mass exchange approach is the result of failure of the guiding system. Lofstrom estimates that failure of the ribbon would release $1.5 \times 10^{15} \mathrm{~J}$, enough to boil 400,000 $\mathrm{m}^{3}$ of water. Since in the authors' opinion catastrophic failure of the device can be expected occasionally, the $2000 \mathrm{~km}$ structure could not be constructed near populated areas. The containment of a metal ribbon moving at hypersonic velocity has yet to be demonstrated at any scale. If this technology can be realized, it will likely find first application as a method of energy storage.

\section{A Freestanding Structure}

We propose an alternative device to provide access to the near-space and space environments that utilises a self-supporting core structure [8]. The structure provides a fixed link between ground and near-space locations enabling the transportation of equipment, personnel and other objects or people to platforms or pods above the surface of the Earth for the purpose of scientific research, communications and tourism. The device may be assembled from the surface upwards, avoiding difficult and expensive inorbit construction. The space-elevator tower can provide access to lower altitude regions and can also be scaled to access altitudes above $15 \mathrm{~km}$, or the typical ceiling altitude for commercial aviation. The approach may be further scaled to provide direct access to altitudes above $200 \mathrm{~km}$ and with the gravitation potential of Low Earth Orbit (LEO) without the technical challenges associated with constructing a cable at least 35,000 km long. The elevator platforms also have significant advantages over orbiting satellite platforms. Geographically fixed but providing access to regions of space closer to the surface than geostationary orbit, elevator platforms provide the ideal means to 
communicate over a wide area and to conduct remote sensing and tourism activities. As a tourist destination, the elevator platforms provide stations located at fixed attitudes from the surface for observation. The elevator platforms provide the means to access safely a region of space with a view extending hundreds of kilometers.

\section{Structural Concept}

The device comprises a pneumatically pressurised core structure consisting of compartments arranged in segments with equipment decks or pods. The compartments are constructed using conventional high stress materials such as Kevlar pressurized with a gas mixture of low atomic mass such as hydrogen or helium. An inertial stabilisation maintains the attitude of the structure with respect to the planet surface using a variety of methods including pressure balancing and angular momentum stabilisation.

Typical core-structure configurations of the elevator are shown in Figure 1. Elevator A has a platform and main pod with segments arranged in a four-segment square configuration with an open lattice structure to brace segments together between decks. Elevator B has a platform and main pod with a segment arrangement of constant exterior diameter and comprising pressurised compartments with decks and pods internal to the segment structure. This option may be desirable from a construction perspective and also if the elevator cars grip the outside diameter of the core. Elevator $C$ has a platform and main pod with a tapered segment configuration and lattice structure incorporating large pods that intersect the segment structure. A $7.0 \mathrm{~m}$ scale model of a structure similar to Elevator A is shown in Figure 2. This 1:2000 scale model comprises three $0.082 \mathrm{~m}$ diameter cores braced at intervals of $1.0 \mathrm{~m}$ (first four intervals) and $1.5 \mathrm{~m}$ (last two intervals). The overall diameter of the structure is $0.34 \mathrm{~m}$. Constructed of laminated polyethylene (Young's modulus of the material measured as $258 \mathrm{MPa}$ ) with an average wall thickness of $0.0013 \mathrm{~m}$, the structure has a total mass of $17 \mathrm{~kg}$ excluding the base support and is freestanding when pressurized with air above 48,000 $\mathrm{Pa}$ (7 PSI).

\subsection{Design Analysis}

The pressure-cell walls consist of a material with very high tensile strength-to-mass ratio, for example, boron or a Kevlar polyethylene composite at a thickness able to retain the 
cell pressure with adequate margins of safety in accordance with engineering practice. Air or gas is used to pressurise the cells using an interlinked network of plumbing such that cells may be repressurised from time to time. At typical surface conditions, atmospheric air has a density of $1.29 \mathrm{~kg} \mathrm{~m}^{-3}$. For a pressurised vessel, the pressure variation with altitude may be derived by consideration of the gravitational force on a unit area air parcel as: $g \rho \partial \mathrm{z}=-\partial p$, where $g$ is the acceleration due to gravity $\left(9.8 \mathrm{~ms}^{-2}\right.$ on Earth), $\rho$ is the mass density of the gas, $p$ is the pressure and $z$ is altitude. At atmospheric pressures the behavior of the gas may be characterized by the ideal gas law as $p=\rho R T / M$, where $R$ is the universal gas constant, $M$ is the molar mass of the gas and $T$ is the temperature in Kelvin. Assuming a constant cell temperature and approximating gravity as constant over altitude, we integrate to obtain the pressure at the top of a cell of altitude $z$ as:

$$
p(z)=p_{0} \exp (-z / H)
$$

where $H=R T / g M$ and is the scale height of the gas column and $p_{0}$ is the base pressure. For Earth’s atmosphere, $H \sim 7.6 \mathrm{~km}$. The load capacity in kilograms of a vertical cylinder of length $l$ and diameter $d$ that has no structural strength under compression is therefore

$$
L=\frac{\pi d^{2} p_{0} \exp (-l / H)}{4 g} .
$$

Assuming the case of a simple single cell structure, the mass of such a segment is given as

$$
m_{\text {element }}=\rho_{A} \pi d l
$$

If the segment is in firm contact with the ground, the apparatus must support only this structural mass as the mass of the pressurization gas may be supported from the base. If the segment is further up the structure, the supporting structure must support the segment mass and the mass of the pressurization gas which is given as:

$$
m_{\text {gas }}=\frac{\rho_{0} b \pi d^{2} H(1-\exp (-l / H))}{4}
$$


where $\rho_{0}$ is the mass density of the gas at one atmosphere and $b$ is the gas pressure at the base in Bar. This expression can also be used to compute the buoyant mass that provides some support for the core. The center of gravity of the support gas is given by:

$$
z_{c o f g}=\frac{H(1-(1+l / H) \exp (-l / H))}{(1-\exp (-l / H))} .
$$

Other gases may be utilized with lower molecular masses than that of air. The mass advantages of other pressurization gases may be approximated by the ratio of their molecular mass with that of nitrogen gas (the dominant constituent of atmospheric air). Thus a structure pressurized with hydrogen will require 28/2=14 times less gas by mass and with helium 28/4=7 times less.

The force required to buckle a simple column similar to elevator structure B under load is given as:

$$
L_{\text {buckling }}=\frac{\pi^{2} E I}{l^{\prime 2}}
$$

where $l$ ' is the effective column length depending on the boundary conditions of the column, $E$ is the effective Young's modulus of the thin-walled column when the core is pressurised and $I$ is the moment of inertia of cross-sectional area. Assuming that the elevator core is fixed at the base and gyroscopically pinned at the top, then $l$ '=l. For a thin-walled circular-section cylinder, $I=\int y^{2} d A=2 \pi t r^{3}$, where $t$ is the thickness and $r$ is the radius.

Consider an example core-structure design for an Earth-based elevator to access near space at $20 \mathrm{~km}$ altitude. Advantageously, to access orbit, the elevator could be constructed at $5 \mathrm{~km}$ altitude in one of four regions on the equator. The core would be required to span a further $15 \mathrm{~km}$ to $20 \mathrm{~km}$ altitude. Based on Elevator B, a suitable structure comprises of gas cells with constant wall thickness $1.2 \mathrm{~cm}$ arranged in a torus of inner diameter $228 \mathrm{~m}$ and outer diameter $230 \mathrm{~m}$. Fabricated from Boron, a $15 \mathrm{~km}$ elevator structure can be supported by 150 bar hydrogen gas. Approximating the structure as two 
concentric cylinders, the mass of the structure is $6.5 \times 10^{8} \mathrm{~kg}$, and the mass of the pressurization gas needed is $1.4 \times 10^{8} \mathrm{~kg}$. Other core designs may be analyzed by comparison with the two-cylinder design and by appropriate adjustment for the amount of wall material utilised.

Constructed at $5 \mathrm{~km}$ altitude, the structure would have a bouyant mass of $3.1 \times 10^{6} \mathrm{~kg}$ giving a total mass of $7.8 \times 10^{8} \mathrm{~kg}$. The load capacity of the structure, in excess of that needed to support itself, is $3.1 \times 10^{8} \mathrm{~kg}$ of force equivalent. The critical buckling load at the top is $4.1 \times 10^{9} \mathrm{~N}$, and at the center of gravity (located at $7.3 \mathrm{~km}$ up the core) the critical load is $1.6 \times 10^{9} \mathrm{~N}$, which exceeds significantly the dead weight load of the building, including the mass of the gas, indicating that the core would be structurally stable and able to support the raising of payloads of mass in excess of $10^{6} \mathrm{~kg}$. By further tapering the wall thickness, further design margin may be obtained by lowering the center of gravity and reducing the structural mass, or taller structures may be constructed. Alternatively, the core diameters can be tapered to increase the structural stiffness in the base, although the variation of core diameter may be undesirable for mounting elevator machinery. Additionally, the core can be segmented and pressurised equivalently without inducing an imbalance of support forces between segment walls. The feasibility of the use of pneumatic beams requires experimental investigation in order to validate theoretical predictions. Zhu, Seth and Quine (2008) examine theoretical predictions compared with experiment for inflated circular section cantilevered beams [9]. Work on the performance of multi-beamed structures is forthcoming.

\subsection{Active Stabilisation System}

In space-elevator configuration, the core structure will be arranged along a linear axis such that the sum of centripetal, gravity and external forces is minimized in the horizontal axes. The weight of the structure and other vertical forces are counteracted by the pneumatic pressure in the cells of the core. Active control machinery is desirable to stabilize the structure against buckling or falling and to couple disturbance torques into other axes. Gyro stablisation is also desirable in order to cause disturbance torques applied to the elevator to induce rotating oscillations in the elevator core rather than longitudinal motions. Figure 3 illustrates a typical modal control strategy. The primary 
control is exerted on the first bending mode of the core structure. An elevator with center of mass at an altitude $h$ under deformation by an external bending moment $B$ must utilise a control law and actuator system in order to adjust the center of mass such that the attitude of the core structure is at an angle $\theta$ to the normal in order to counteract the disturbance. Other structure-bending moments do not displace substantially the center of mass of the core structure. Consequently, the core structure can be controlled independently by the variation of segment pressures along the core at a wavelength and period characteristic of the bending mode. Conveniently, an extended Kalman filter that does not require the computation of model derivatives [10] may be utilized to predict the amplitude and phase of the bending modes from nonlinear models developed to describe structural behaviour. Geodetic sensors measure the precise orientation of the elevator structure and its rate of change. Measurements of cell pressure and load are also supplied to the estimator that generates a real-time estimate of system state. Additionally wind loads and other forcing terms are measured in order to improve the dynamic response. The presence of a gyroscopic stabilisation system will cause an oscillation $\omega$, which is beneficial to control as it provides efficient energy storage of time-varying torques $\mathrm{B}$ as shown in Figure 3; oscillations may be damped by pneumatic dampers that vary the compartment pressures at the core-structure harmonic frequencies so as to dampen oscillations actively. The energy that may be extracted by damping is given as the product of rate of change of pressure and sum of the compartment volumes. The control system typically acts on the first six bending moments of the core or until the structural bending modes may be neglected. The damping effect may be achieved actively using a high pressure line-and-vent network system and passively by allowing support gas to vent from compartment to compartment along a connecting line network. For the primary bending mode the force exerted transverse to the core structure is $m g \sin \theta$. Consequently, for a building lean angle of $1.0^{\circ}$ the force is $1 / 57$ of the weight force. Pearson anticipates a peak wind velocity of $150 \mathrm{~ms}^{-1}$ giving a typical dynamic pressure of $8300 \mathrm{Nm}^{-2}$ applied over a $3 \mathrm{~km}$ vertical interval. For our $15 \mathrm{~km}$ structure, this corresponds to a total dynamic pressure force of 3.0x10 $\mathrm{N}$ applied at approximately $1.5 \mathrm{~km}$ above the structure base. Assuming weight force is the sole means of control stabilization, the center of gravity of the structure ( $7.4 \mathrm{~km}$ above the base) would need to be inclined at $2.0^{\circ}$ from the vertical 
in order to counter this force. Located at $5 \mathrm{~km}$ altitude, it is extremely unlikely that the structure would encounter such a large disturbance in the real world. The maximum control forces that must be exerted to stabilize the core structure are determined by the lean angle error in the primary bending mode as tabulated in Table 1 . Therefore, if the attitude error of the core structure can be maintained at less than $0.1^{\circ}$ then the maximum reaction force required from the active control system is $1.3 \times 10^{7} \mathrm{~N}$ (or 1400 tonnes of mass equivalent force).

\subsection{Compressor Machinery}

Unless cells are pre-pressurised and in case of leakage, the compressor stations are required to pump gas into the elevator. Compressor stations may be sized by predicting and monitoring compartment leak rates with time and including margin for tower pneumatic control. Alternatively, a high pressure gas line may be utilised to pressurise and control the core structure with compressor machinery and pressure reservoirs mounted on the ground.

\subsection{Gyro-Control Machinery}

Control machinery may be located along the elevator in substantial contact with the core. The control machinery consists of a set of one or more heavy spinning wheels or gyroscopes that increase the angular momentum of the elevator's core structure in order to stablise its orientation in space. Conveniently, the wheels may also be adapted to act as compressors and pressurise the structure. The wheels are spun at high radial velocities in order to ensure that a significant fraction of the structure's angular momentum is stored in their motion. The machinery normally operates continuously while the structure is in operation and is duplicated so as to ensure redundancy and downtime for maintenance access. The wheels may also be installed inside the pressurized cells so as to induce vortices in the support gas to further enhance the gyroscopic mass; however, this process may not be very energy efficient. The minimum wheel speed required to completely stabilize a spinning gyroscope with respect to gravity is given as: 


$$
\omega_{\min }=\sqrt{\frac{4 m g l I_{x}}{I_{z}^{2}}},
$$

where $m g$ is the weight of the core structure, $l$ is the length of the core structure, $I_{x}$ is the inertia moment and $I_{z}$ the polar inertia moment of the cross-section of the core structure, respectively.

For realistic wheel-speed solutions, cores may only be completely stabilized up to $150 \mathrm{~m}$; however, the stabilization effect is still useful. In a single wheel design, the wheel would be orientated to spin with its axis aligned with the elevator core such that horizontal forces applied to the core were transferred to processional motions in the core.

The control machinery also includes active damping systems that enhance the structure's ability to damp oscillations by leaking air from cell to cell using a control valve network. This machinery may be controlled and powered by pneumatic or electrical means as is convenient and can provide a means to communicate with elevator components. A typical segment configuration is shown in Figure 4. The segment consists of a closed circular compartment network with internal gyroscopic stabilization wheels and control and compressor machinery supported by pressurization gas in compartment columns. The segment is supplied with resources including gas and power along conduits mounted between pressure decks. The gyro wheels are encapsulated in separate sections so that they may be run under near-vacuum conditions.

\subsection{Construction}

Figure 5 illustrates one construction method where the core is erected vertically using a mechanism that extrudes or attaches elevator segments. Pods containing control and stabilization machinery are embedded in the structure as it is extruded by a roller system from a stack of similar pod units. The segment walls and pressure compartments are formed as an extrusion molding of a liquid core material. A winding mechanism also located with the extrusion molding system embeds fibers into the core structure in order to increase the elastic resistance of the structure. Pneumatic pressure and the roller mechanism raise and lower core sections and the entire elevator structure. 
Figure 6 shows an alternative construction approach where sections are raised by means of a climbing construction elevator that grips the external surface of the existing structure as it raises and installs segments section by section. Advantageously, segments equipped with stabilisation systems may be energised by means of an umbilical connector such that the new section may be raised completely above the construction elevator and installed on the existing core structure by means of a horizontal track installed on the top of the construction elevator. The center of gravity of the combined system is adjusted actively during the section installation in order to maintain it over the core's surface footprint and to provide support for the structure in the presence of external disturbance torques. The use of gyro stabilization during construction would be desirable as sections up to $150 \mathrm{~m}$ may be balanced on a pivot mount.

\subsection{Maintenance and Decommissioning}

For reliability and repair, a multi-core segmented structure is desirable in order to ensure that elevator integrity can be maintained during maintenance of cells and to facilitate leak repair. Failure tolerance can be enhanced by the duplication of subsystems used in other high technology systems, with critical systems such as compressors and gyro-stabilization wheels operated in hot redundancy mode. A segmented core structure also enables the disassembly of the system during decommissioning and enables the core structure to be dismantled in a top-down process while power and pressure are maintained to the remaining core structure and systems.

\subsection{Operation}

A platform or pod supported by the space-elevator tower has significant advantages over orbiting satellite platforms. Geographically fixed but providing access to regions of space closer to the surface than geostationary orbit, elevator platforms provide the ideal means to communicate over a wide area and to conduct remote sensing and tourism activities. As a tourist destination, the elevator platforms provide stations located at fixed altitudes from the surface for observation. The elevator platforms provide the means to access a region of space safely with a view extending hundreds of kilometers. Small systems may be mobile and delivered to sites for temporary applications such as the provision of 
temporary communications towers typically between $25 \mathrm{~m}$ and $150 \mathrm{~m}$. Elevator cars enable equipment to be accessed and maintained during operation.

For a spherical planet of radius $r$, and elevator of height $h$, the horizon is at a surface distance $s$ of:

$$
s=r \arccos \left(\frac{r}{r+h}\right)
$$

assuming a level surface and neglecting diffraction. This corresponds to a line of sight that is horizontal at the horizon. As an example, on Earth $(r=6380 \mathrm{~km})$ an elevator extending $20 \mathrm{~km}$ above the surface will have a view extending $500 \mathrm{~km}$ and access a surface area of $780,000 \mathrm{~km}^{2}$. For a line of sight with an elevation of $\alpha$ radians above the horizon the geometry is more complex but can be recast in terms of the height of elevator required as:

$$
h=\frac{r}{\cos (s / r)}-r+\frac{r \tan (s / r) \sin \alpha}{\sin (\pi / 2-s / r-\alpha)} \text {. }
$$

Thus an elevator of height $20 \mathrm{~km}$ will have a line of sight greater than $10^{\circ}$ elevation at surface distances of up to a range of $108 \mathrm{~km}$ and access an area more than 36,000 $\mathrm{km}^{2}$.

\subsection{Utility for Space Launch}

Although ascending to an altitude significantly below 35,000 km will not place a payload directly into Earth orbit, a platform or pod supported by the space-elevator tower has significant advantages over a surface-based launch platform. While surface-based rockets must be designed to overcome atmospheric air resistance, launch from a high-altitude platform has no such requirement, and, consequently, existing space equipment such as an orbital transfer stage or conventional upper stage can be used to insert payloads directly into Earth orbit. Ideally, payloads should be raised to the highest feasible altitude before launching in order to maximize the energy advantages; however, the energy advantages for space flight are readily leveraged above $5 \mathrm{~km}$. The energy required for a surface launch to Low Earth Orbit can be expressed per kilogram of payload as the sum of potential and kinetic terms as: 


$$
E_{\text {Orbit }}=g h_{1}+\frac{1}{2}\left(v-v_{0}\right)^{2}
$$

where $h_{1}$ is the altitude of the orbit, $v$ is the orbital velocity and $v_{0}$ is the initial velocity (assuming this is in the same direction of motion). Here, we have assumed conservatively that $g$ is constant with altitude; however, for a typical LEO orbit altitude ( $\left.h_{1}=300 \mathrm{~km}\right)$, $g=0.9 g_{0}$ where $g_{0}$ is the surface gravity. By inspection, the energy required is approximately $1 \mathrm{MJ}$ per $100 \mathrm{~km}$ of orbital altitude in potential energy and $27 \mathrm{MJ}$ in kinetic energy assuming a $7.9 \mathrm{kms}^{-1}$ orbital speed and an energy efficient equatorial launch. For a space elevator, the energy required is:

$$
E_{\text {Orbit }}^{\prime}=g\left(h_{1}-h\right)+\frac{1}{2}\left(v-v_{0}-\phi h\right)^{2}
$$

where $\phi$ is the angular rate of rotation of the planet $\left(\phi=7.27 \times 10^{-5}\right.$ rads $^{-1}$ for Earth) and assuming an equatorial surface site. For a $20 \mathrm{~km}$ elevator, the energy requirement is reduced by approximately $0.2 \mathrm{MJ}$; for a $300 \mathrm{~km}$ structure, the energy is reduced by $3 \mathrm{MJ}$. This net reduction in payload energy appears small; however, rocketry is an extremely inefficient means of providing payload energy, particularly during the early phase of spaceflight as we now discuss.

Consider a rocket of mass $m$ launched vertically in order to escape the lower atmosphere. The equation of motion is:

$$
m a=\dot{m} v_{p}-m g
$$

where $a$ is the acceleration of the mass, $\dot{m}$ is the rate of use of fuel mass and $v_{p}$ is the exit velocity of the propellant. In order to simply hover ( $a=0)$, the mass fraction of fuel that must be expelled is:

$$
\left(\frac{\dot{m}}{m}\right)=\frac{g}{v_{p}}=\frac{g}{g I_{s p}}=\frac{1}{I_{s p}},
$$

where $I_{s p}$ is the specific impulse of the rocket fuel in seconds. Consequently, a typical biopropellant system $\left(I_{s p}=300 \mathrm{sec}\right)$ must burn $1 / 300$ of the rocket mass in fuel per second 
of flight in order simply to hover. In order to overcome this loss, the rocket must gain velocity tangential to the surface as rapidly as possible. This has the effect of reducing the effective gravity that must be overcome as:

$$
g^{\prime}=\frac{r^{2} g_{0}}{(r+h)^{2}}-\frac{v_{t}^{2}}{(r+h)}
$$

This reduction can be tabulated, as shown in Table 2. Thus for tangential velocities less than $4 \mathrm{kms}^{-1}$, the reduction in gravitational acceleration is less than $25 \%$. If we now consider a typical launch scenario and flight to $20 \mathrm{~km}$, we may compare the energy used to obtain an efficiency ratio for the rocket and, consequently, determine an overall energy saving by employing a space elevator for the initial ascent. The rate of energy dissipation by the rocket is given as the kinetic energy imparted to the fuel as:

$$
\dot{E}=\frac{1}{2} \dot{m} v_{p}^{2}=\frac{m(a+g)}{2} v_{p}
$$

Consider a typical launch with $a=3 g$ and fuel specific impulse $I_{s p}=300$ sec. If we assume the early flight to $20 \mathrm{~km}$ is almost vertical, the time to ascend to $20 \mathrm{~km}$ is $t=\sqrt{2 h / a}=36.9 \mathrm{sec}$, and the vertical velocity is $1.1 \mathrm{kms}^{-1}$. If we assume that the mass of the rocket is 20 times that of the payload $(m=20)$ then in this example

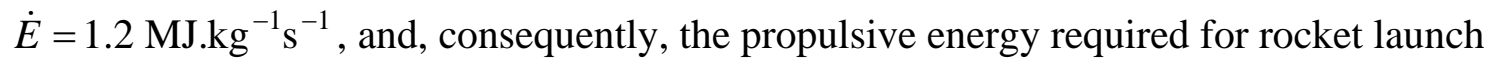
to $20 \mathrm{~km}$ is approximately $43 \mathrm{MJ} \mathrm{kg}^{-1}$ while the gain in kinetic energy by the payload is $0.6 \mathrm{MJ}$ and the gain in potential energy is $0.2 \mathrm{MJ}$. The efficiency of the rocket in achieving useful work on the payload is therefore $0.8 / 43=0.0186$ or $1.86 \%$ during this flight phase. Assuming staging occurs after $20 \mathrm{~km}$ altitude, the rocket mass at $20 \mathrm{~km}$ is $\exp (-4 / 300 * 36.9)=0.61$ and hence approximately $39 \%$ of the launch mass in fuel must be consumed.

In contrast, the work efficiency of a well designed electric elevator $(m=1.5)$ may approach 30\% with primary losses in power conversion and the gravitational potential gained by the elevator car. The efficiencies may be higher if the car's potential energy is recovered during descent. Although the efficiency gains are only applied to a small fraction of the total energy needed for launch, the use of a space elevator avoids the 
vertical ascent phase while the rocket escapes the atmosphere, and little horizontal velocity is gained in order to counteract gravity. The elevator launch advantage may be examined by simple numerical simulation to compare launch from $20 \mathrm{~km}$ with launch from the ground. Consider a highly simplified scenario where a single stage-to-orbit rocket is launched to a typical circular orbital height of $120 \mathrm{~km}$. Using equations 12 and 14 and assuming an adiabatic approximation for atmospheric density that contributes a drag term proportional to the square of velocity, a two-dimensional motion model may be developed to simulate the rocket-flight path using simple Newtonian equations of motion. Figure 7 shows two typical flight profiles designed to achieve orbital velocity by $120 \mathrm{~km}$ altitude computed with a simulation time step of $0.05 \mathrm{sec}$. Both assume a constant $3 g$ acceleration in the direction of motion and an initial rocket of mass $10^{6} \mathrm{~kg}$ that carries a fuel with specific impulse $I_{s p}=250 \mathrm{sec}$. For both launch cases, an optimal initial launch angle was determined experimentally and fuel use, final altitude, final speed and time to orbit were computed as shown in Table 3. While actual rocket-launch profiles are more complicated because of staging, thrust vectoring and maneuvering, this simple example serves to contrast launch efficiency. Comparing initial to final rocket-mass ratios, the elevator launch at $20 \mathrm{~km}$ is shown to be $26 \%$ more efficient than the equivalent ground launch, and the flight from the elevator is 19 seconds shorter. This advantage may be leveraged as a reduction in initial rocket size or an increase in payload capability.

\section{Conclusions}

Although the challenges of constructing space elevators are immense, we describe a practical concept for the construction of a device to access altitudes above $20 \mathrm{~km}$ that is realisable using current material technologies. The suborbital tower approach also has many advantages over more traditional means of accessing space. Wide area, bidirectional communications grids can be established over vast areas without the dataleniency delay caused by transit time to and from geosynchronous orbit. For space tourism, the suborbital tower offers the thrill of ascending into space without the risks and environment effects associated with conventional rocketry. Suborbital towers may also facilitate construction of geoscynchronous space tethers, providing an idea surface 
mounting point where an orbital tether could be attached without experiencing atmospheric turbulence and weathering in the lower atmosphere.

\section{References}

[1] Clarke A. C. The Fountains of Paradise. Harcourt Brace Jovanovich, N.Y. Gollancz, London, 1978.

[2] Pearson J, The orbital tower: a spacecraft launcher using Earth's rotational energy, Acta Astronautica, 2, 785-799, 1975.

[3] Edwards B. C., Design and deployment of a space elevator, Acta Astronautica, 2, 785-799, 2000.

[4] Boyd R. R. and D. D. Thomas, Space Elevator, US Patent 6491258, Filing date: Dec 6, 2000, Issue date: Dec 10, 2002, (Assignee: Lockheed Martin Corporation).

[5] Dempsey, J. G. System and method for space elevator, US Patent 6981674, Filing date: Sep 16, 2004, Issue date: Jan 3, 2006.

[6] Pugno N., On the strength of the carbon nanotube-based space elevator cable: from nanomechanics to megamechanics, J. Phys.: Condens. Matter, 18, S1971S1990, 2006.

[7] Lofstrom K. H., The Launch Loop: A low cost earth-to-high-orbit launch system, AIAA-85-1368, 21 ${ }^{\text {st }}$ joint propulsion conference, 1985.

[8] Quine B.M, Space Elevator, United States Provisional Patent No. 60/890,947, Feb 21, 2007.

[9] Zhu Z. H., Seth R. K., and B. M. Quine, Experimental Investigation of Inflatable Cylindrical Cantilevered Beams, JP Journal of Solids and Structures, vol. 2, issue 2, 95-110, 2008.

[10] Quine B. M., A derivative free implementation of the extended Kalman filter, Automatica, 42, 1927-1934, 2006. 


\section{Figures}

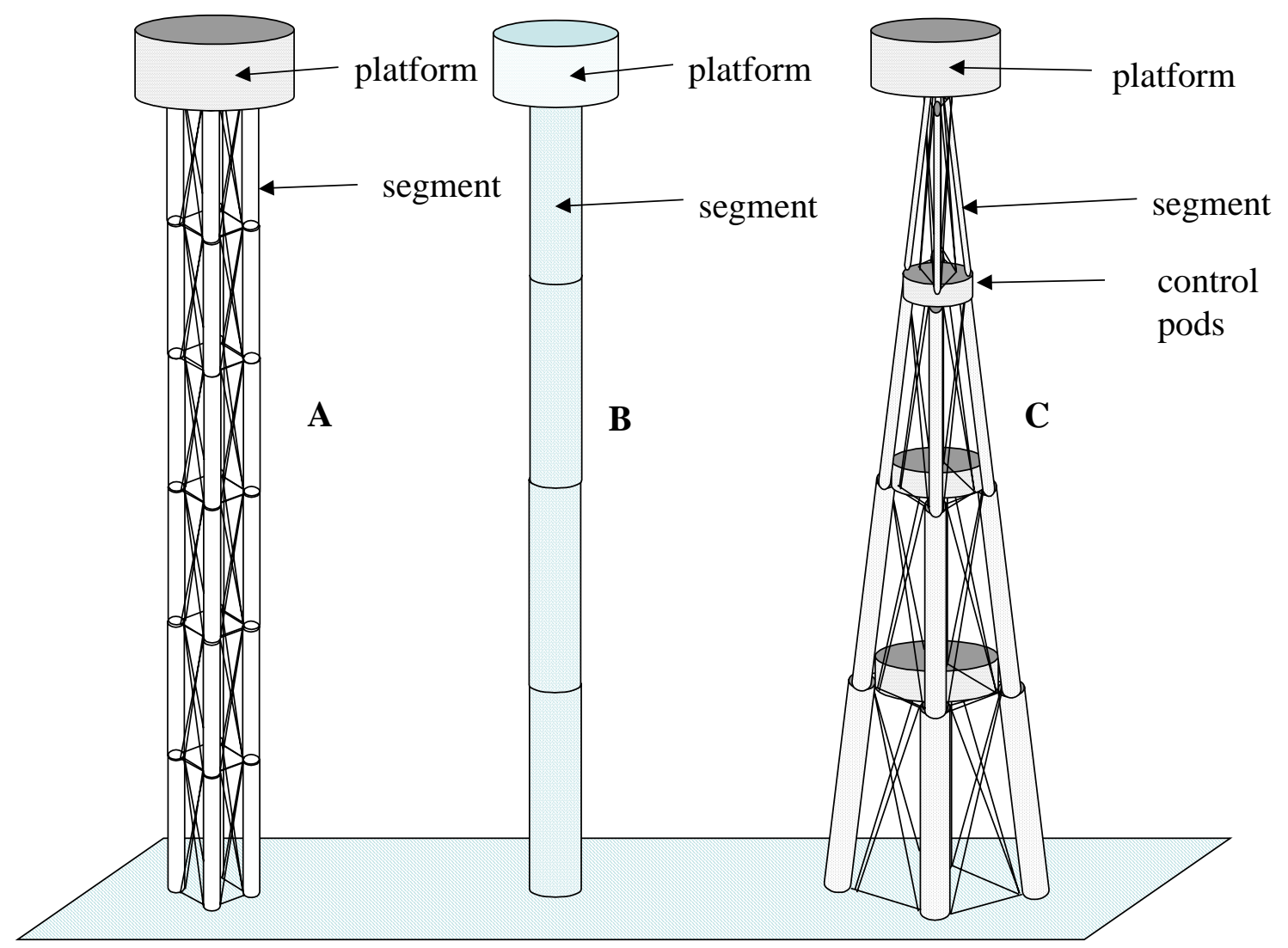

Figure 1: Core-structure configurations (A, B and C). 


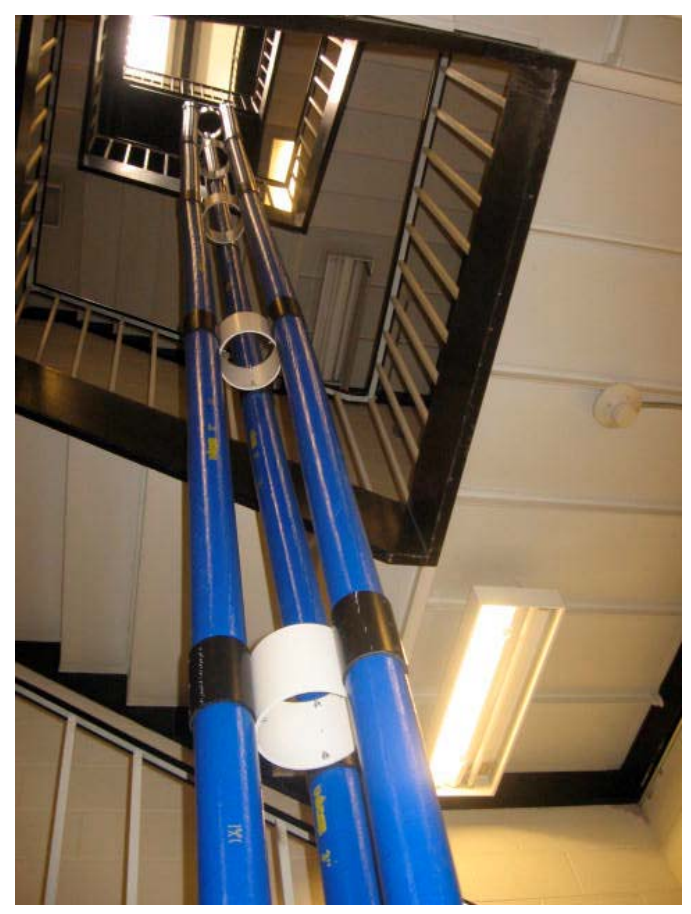

Figure 2: $7.0 \mathrm{~m}$ demonstration device installed in stairwell. 
Quine, Seth and Zhu

Page 22

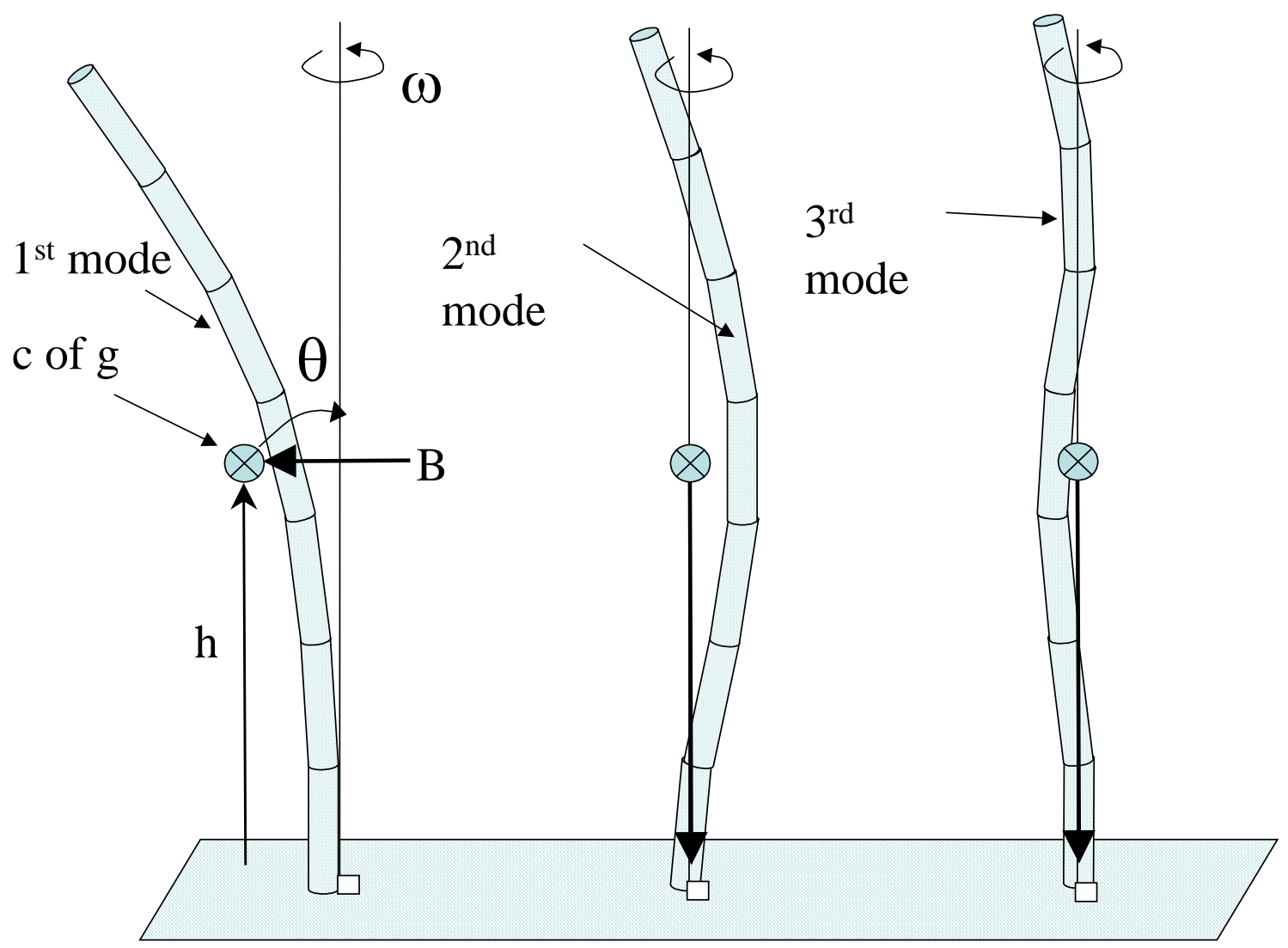

Figure 3: Elevator structure primary bending modes. 


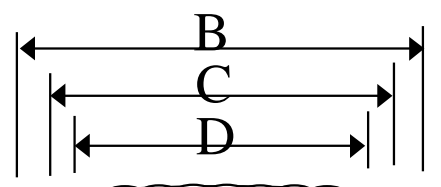

closed circular
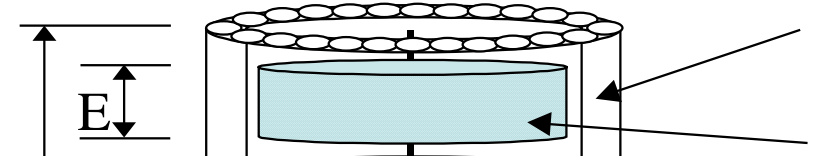

compartment network

gyroscopic stabilization wheels

control and compressor machinery

pressurized compartment columns

A gas and power conduits

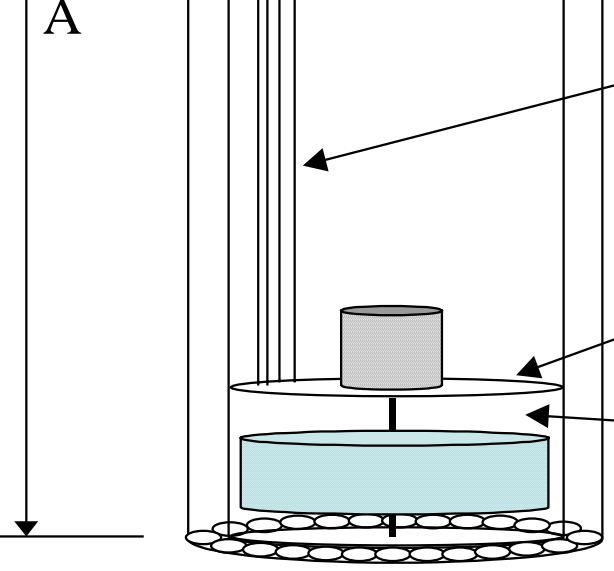

Figure 4: Typical segment assembly with internal machinery pods. 


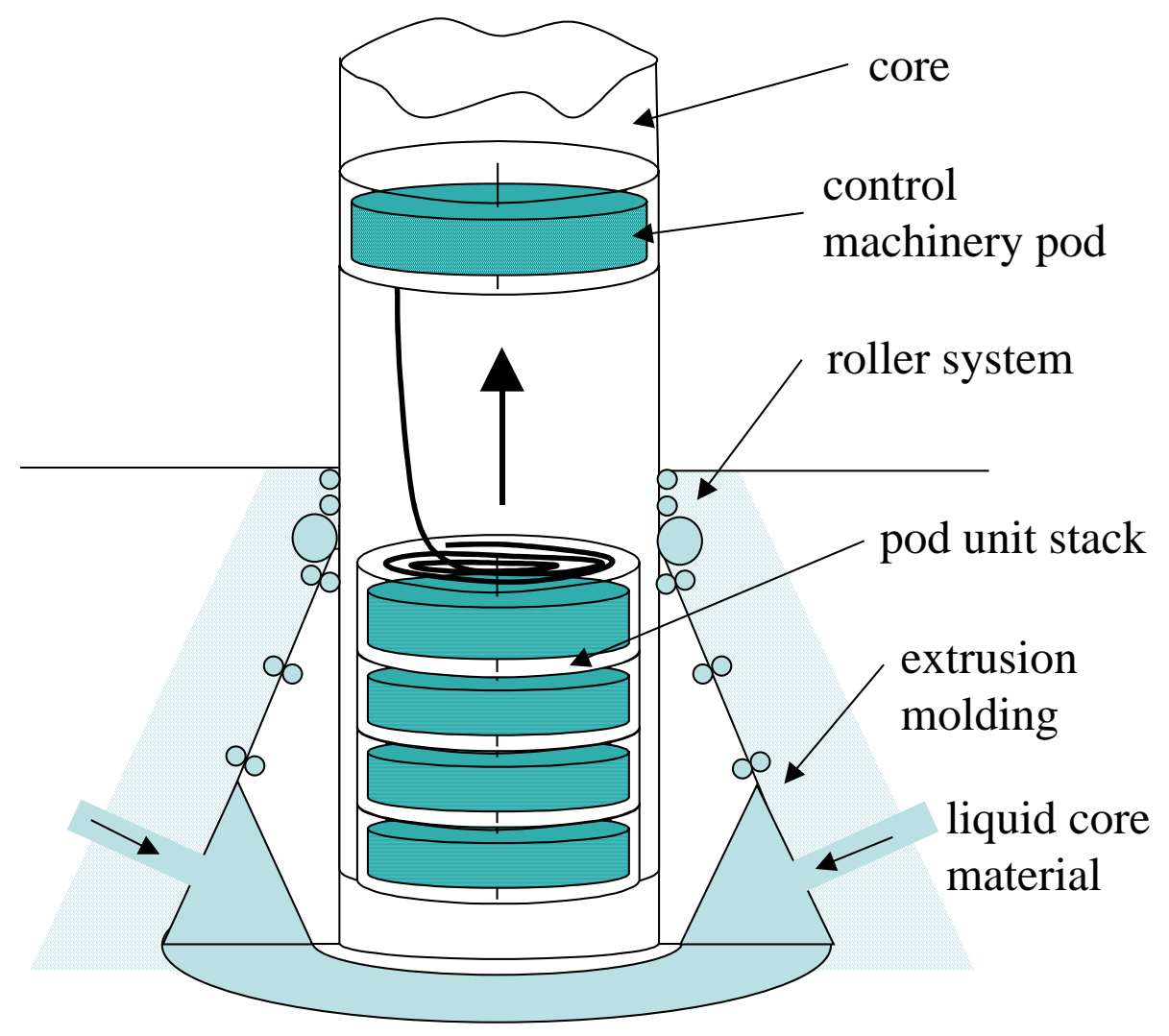

Figure 5: Construction of core by extrusion method. 
Quine, Seth and Zhu

Page 25

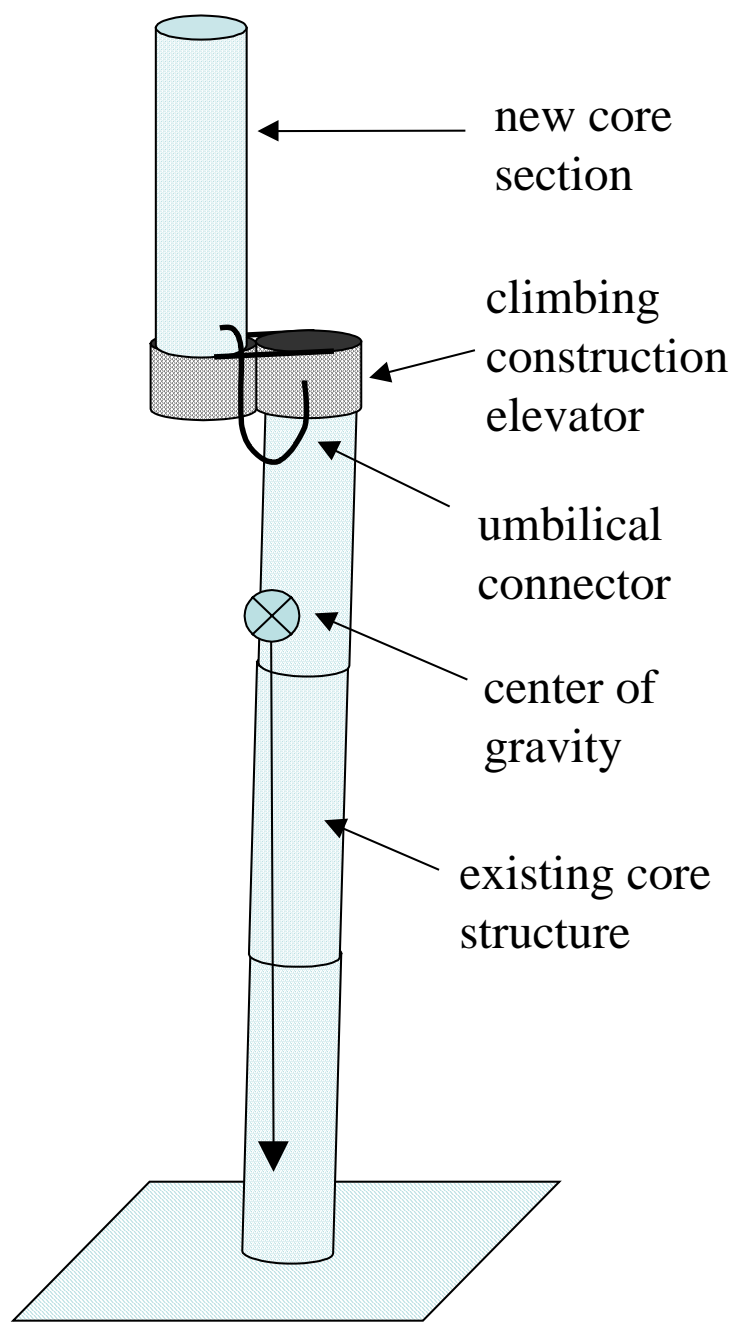

Figure 6: Construction of core section by section using construction elevator apparatus. 


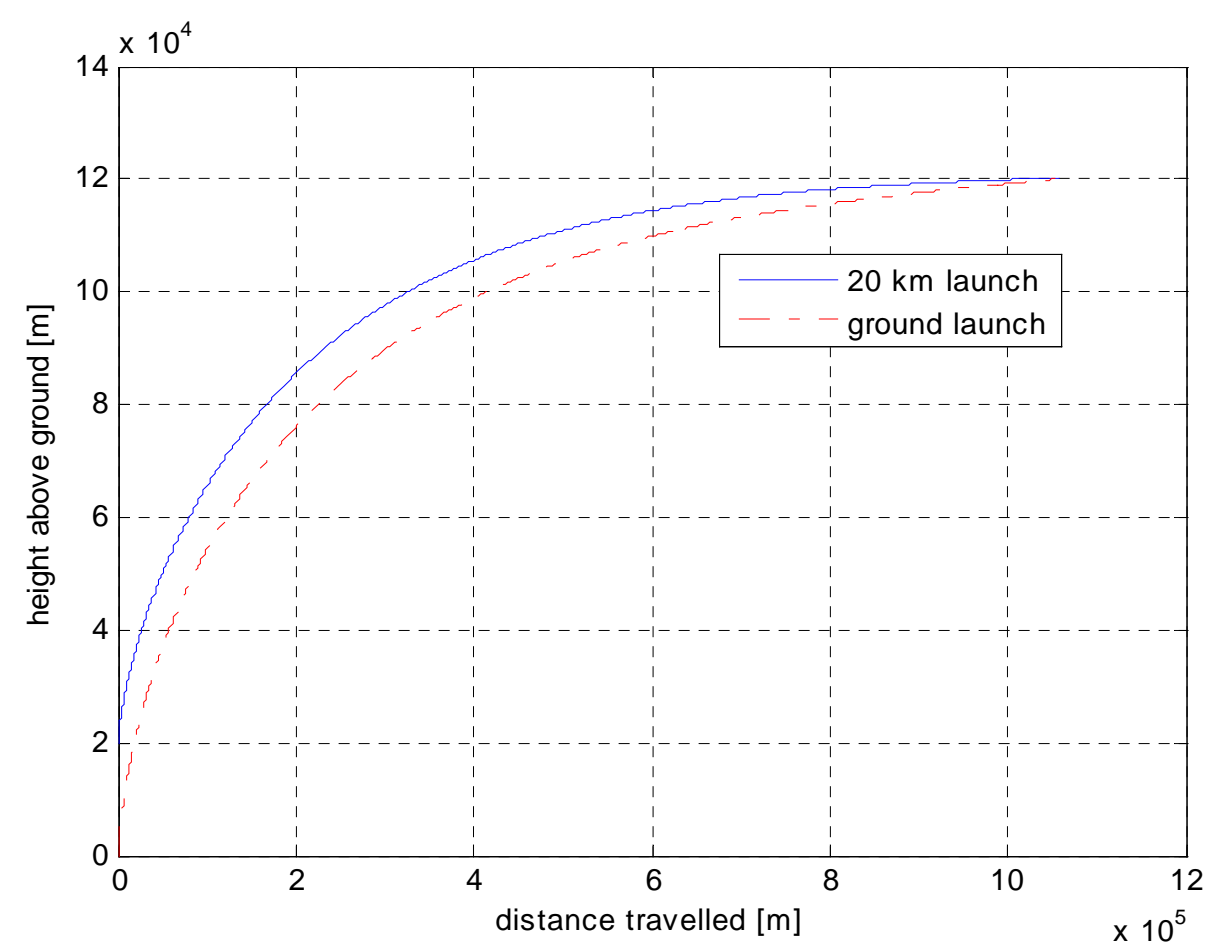

Figure 7: Ground and elevator launch (initial altitude of $20 \mathrm{~km}$ ) comparison.

\section{Tables}

Table 1: Control Performance tradeoff.

\begin{tabular}{|l|l|l|}
\hline $\begin{array}{l}\text { Lean Angle } \\
\text { Error } \\
\text { [degrees] }\end{array}$ & $\begin{array}{l}\text { Control Force } \\
\text { (Fraction of } \\
\text { weight) }\end{array}$ & $\begin{array}{l}\text { Control Accuracy } \\
\text { [meters] }\end{array}$ \\
\hline 1 & $1 / 57$ & 122 \\
\hline 0.1 & $1 / 572$ & 12.2 \\
\hline 0.01 & $1 / 5729$ & 1.2 \\
\hline
\end{tabular}


Table 2: Effective gravity.

\begin{tabular}{|c|c|c|}
\hline $\begin{array}{c}\text { Tangential Velocity } \\
v_{p}\left(\mathrm{kms}^{-1}\right)\end{array}$ & $\begin{array}{c}\text { Effective } \\
\text { Gravity } g^{\prime} \\
\left(\mathrm{ms}^{-2}\right)\end{array}$ & $\begin{array}{c}\text { Reduction over } \\
\text { surface gravity } \\
\left(\mathrm{ms}^{-2}\right)\end{array}$ \\
\hline 1.0 & 9.58 & 0.22 \\
\hline 2.0 & 9.11 & 0.69 \\
\hline 3.0 & 8.33 & 1.47 \\
\hline 4.0 & 7.24 & 2.56 \\
\hline 7.0 & 2.08 & 7.71 \\
\hline
\end{tabular}

Table 3: Space Elevator Launch vs. Ground Launch.

\begin{tabular}{|c|c|c|}
\hline Parameter & $\begin{array}{c}\text { Elevator } \\
\text { Launch } \\
(20 \mathrm{~km})\end{array}$ & $\begin{array}{c}\text { Ground } \\
\text { Launch }\end{array}$ \\
\hline $\begin{array}{c}\text { Initial launch } \\
\text { elevation (deg) }\end{array}$ & $86.9^{\circ}$ & $87.8^{\circ}$ \\
\hline Final altitude (m) & 120270 & 120210 \\
\hline Final speed (m/s) & 7835 & 7836 \\
\hline $\begin{array}{c}\text { Final rocket mass } \\
\text { (kg) }\end{array}$ & 14300 & 10500 \\
\hline $\begin{array}{c}\text { Final mass fraction } \\
\text { of launch mass }\end{array}$ & 0.0312 & 0.0248 \\
\hline Time to orbit (sec) & 289 & 308 \\
\hline
\end{tabular}

